Revista de Investigación Educativa 25

julio-diciembre, 2017 | ISSN 1870-5308 | Xalapa, Veracruz

Instituto de Investigaciones en Educación | Universidad Veracruzana

\title{
Autopercepción de habilidades de aprendizaje en ambientes virtuales
}

Self-perception of learning skills in virtual learning environments

\author{
Mtra. Ana María Orozco Santa María \\ Docente \\ Universidad Anáhuac Querétaro, México \\ anaor8555@gmail.com
}

Dra. Ma. Teresa García Ramírez

Coordinadora de Maestría Universidad Autónoma de Querétaro, México

teregar@uaq.mx

Las universidades agregan nuevos programas semipresenciales o totalmente en línea y los estudiantes se enfrentan a un aprendizaje en solitario que requiere de habilidades que son más necesarias en dichas modalidades. Con esta idea, el propósito del presente trabajo fue explorar la percepción que tienen estudiantes universitarios matriculados en asignaturas mediadas por tecnología sobre sus habilidades para ser individuos digitalmente alfabetizados. Se realizó una investigación con alcance descriptivo-cuantitativo en la Universidad Autónoma de Querétaro. Se utilizó como técnica de recolección de datos una encuesta enfocada en conocer la autopercepción sobre habilidades que se requieren para el aprendizaje en ambientes virtuales. El estudio mostró que existen diferencias significativas entre habilidades y que los niveles más bajos se ubican en el aprendizaje autónomo y el uso de herramientas Web 2.o. Se concluye que la inclusión de estrategias instruccionales que desarrollen las diferentes dimensiones de alfabetización digital es esencial para instruir estudiantes.

Palabras clave: Alfabetización digital; ambientes virtuales de aprendizaje; habilidades. 
Universities add new blended or fully online programs and students face a solo learning that requires skills that are most needed in these modalities. With this in mind, the purpose of this study was to explore the perception that university students enrolled in technology mediated courses have about their skills to become digitally literate. An investigation was conducted with quantitative descriptive scope at a public university in Queretaro. A survey was used as data collection technique focused to determine the self- perception about skills required for learning in virtual environments. The study showed that there are significant differences among skills and that the lowest levels are located in autonomous learning and use of Web 2.0 tools. It is concluded that the inclusion of instructional strategies that develop the different digital literacy dimensions is essential to instruct students.

Keywords: Digital literacy; virtual learning environments; skills.

\title{
Autopercepción de habilidades de aprendizaje en ambientes virtuales
}

\author{
Self-perception of learning skills in \\ virtual learning environments
}

\section{Introducción}

$\mathrm{E}^{\mathrm{n}}$ n la actualidad, la oferta educativa de las universidades en México agrega cada ciclo escolar nuevos programas semipresenciales o totalmente en línea que utilizan diferentes Sistemas de Gestión de Aprendizaje, conocidos en inglés como Learning Management Systems (LMS). Los LMS fueron creados para ofrecer una opción flexible de aprendizaje, se encuentran en la web y han sido diseñados para ofrecer una dimensión tecnológica que está constituida por las herramientas o aplicaciones informáticas, y una dimensión educativa, que aloja en su interior los elementos necesarios para desarrollar un proceso de enseñanza-aprendizaje.

Los LMS ofrecen diferentes maneras de manejar contenidos y materiales para desarrollar en los estudiantes conocimientos, habilidades y actitudes. A pesar de la 
gama de opciones para presentar contenidos, las lecturas siguen siendo el principal medio para transmitir información, la cual debe ser procesada y asimilada por los estudiantes para apropiarse de nuevos conocimientos. Los estudiantes deben leer textos de todo tipo alojados en los LMS y diariamente se enfrentan a un proceso de aprendizaje autónomo que implica una participación más activa.

Con el aumento de cursos semipresenciales o totalmente en línea, los estudiantes hacen frente a un aprendizaje en solitario que requiere atributos y habilidades distintos a los que usan en la enseñanza presencial. Entre ellos están la destreza para el manejo del tiempo, la planificación anticipada, la capacidad para comunicarse de manera eficaz, la capacidad para hacerse responsable del propio aprendizaje y el uso de la tecnología (Talbot, 2003).

Los estudiantes a distancia, usuarios de los LMS, deberán superar sus propias actitudes hacia el estudio, como apatía, falta de cultura general, carencia de estrategias de aprendizaje y equilibrio emocional, que afectan el rendimiento académico (Amaya \& Prado, 2002).

Las problemáticas señaladas requieren de la atención de todos los involucrados en el diseño e implementación de cursos en línea para lograr mejores aprendizajes en los estudiantes. Además de un sólido diseño instruccional se requiere que docentes y estudiantes cuenten con un repertorio de estrategias de diferentes tipos que estén alojadas en herramientas Web 2.0 y que cumplan dos funciones: promover el conocimiento de tecnologías y desarrollar diferentes habilidades.

Estéfano (2013) señala que uno de los objetivos de la educación a distancia $(\mathrm{EaD})$ es "proveer métodos de enseñanza mediante el manejo de diversas estrategias para promover en el estudiante la reflexión, el análisis y el aprendizaje autónomo" (p. 23). Considerando lo dicho por Estéfano, conocer lo que perciben los estudiantes sobre sus habilidades para aprender proveerá datos para establecer acciones encaminadas a la alfabetización digital. Con dicha información, se podrán programar actividades que desarrollen habilidades para el estudio y uso de la tecnología con fines educativos.

En consecuencia, la pregunta de investigación que orientó el presente trabajo fue: ¿cómo perciben los estudiantes universitarios matriculados en cursos mediados por tecnología las habilidades requeridas para ser individuos digitalmente alfabetizados? 


\section{Antecedentes y fundamentos teóricos}

Hernández y Díaz Barriga (2013) mencionan que vivimos en una sociedad inundada de información que no ha sido filtrada y que estamos poco preparados para convertirla en conocimiento valioso. Señalan también que los estudiantes no reciben la formación requerida para un aprendizaje reflexivo y crítico.

Aprender usando la tecnología requiere que los alumnos desarrollen habilidades para el autoaprendizaje, el sentido crítico y la búsqueda responsable de información. Así mismo, deben tener un dominio de la comprensión lectora textual, audiovisual y multimedia y ser capaces de expresarse y comunicarse (Bartolomé \& Grané, 2004). La letra digital, al igual que la letra escrita, requiere la puesta en marcha de diferentes conocimientos y habilidades que el auto-lector digital debe aprender, ya que no se obtienen de forma espontánea (Hernández, Ramírez \& Cassany, 2014).

Alfabetizar digitalmente "es un proceso más complejo que la mera capacitación en el manejo del hardware y del software” (Area, Moreira \& Vidal, 2012, p. 25). Un modelo integrado y global de alfabetización digital debe desarrollar diferentes dimensiones competenciales: instrumental, cognitivo-intelectual, sociocomunicacional, axiológica y emocional. A fin de desarrollar dichas dimensiones, las habilidades para la comprensión lectora, la comunicación escrita, el aprendizaje autónomo y el uso de herramientas Web 2.o, son fundamentales para que los estudiantes analicen e interpreten información, se comuniquen por escrito usando diferentes herramientas y tengan la capacidad de aprender por sí mismos.

La diversidad que existe entre estudiantes puede explicarse por el enfoque hacia el aprendizaje y la orientación hacia el estudio. Los estudiantes con una orientación hacia la reproducción tienen un acercamiento superficial al aprendizaje; aquéllos con una orientación con sentido utilizan un enfoque más profundo que los lleva a explorar la aplicación de los nuevos conocimientos y, por último, los que tienen una orientación al logro, usan un enfoque estratégico que combina lo superficial y lo profundo según sea requerido (Felder \& Brent, 2005). Estas diferencias estratégicas que existen entre estudiantes constituyen una poderosa razón para identificar lo que conocen sobre sus habilidades para aprender en ambientes virtuales, y así, orientar el diseño instruccional hacia actividades que permitan alfabetizarlos digitalmente y producir aprendizajes que van más allá de la simple memorización.

La capacidad cognitiva del alumno es el eje para la toma de decisiones instruccionales y educativas que deberán privilegiar una participación activa (Hernández, 
1998). La concepción del alumno como sujeto activo procesador de información del paradigma cognitivo constituye un aspecto básico para el diseño de actividades usando estrategias que permitan desarrollar diferentes habilidades.

Dewey (1989) consideraba que "la información es una carga indigesta, a menos que se le entienda” (p. 82). La analogía que hace Dewey permite reflexionar sobre la importancia de las habilidades de aprendizaje para facilitar una participación activa en el estudio. Para entender los contenidos alojados en los LMS es fundamental que los estudiantes realicen actividades de abstracción, elaboración, esquematización, organización, entre otras (Tuckman \& Monetti, 2011). Los LMS cuentan con herramientas de comunicación y colaboración, de gestión de contenidos y de gestión de grupos que permiten dar soporte al proceso de enseñanza aprendizaje y ponen a disposición de los estudiantes recursos que promueven la interactividad (Fernández \& Rivero, 2014).

La tarea del aprendizaje implica una participación activa del estudiante en la que debe seleccionar, organizar y comprender los contenidos de una materia para crear estructuras de conocimiento que relacionen lo nuevo con los conocimientos previos, obteniendo un aprendizaje significativo (Díaz Barriga \& Hernández, 2002).

Isman, Altinay y Altinay (2004) mencionan en su investigación que para que los alumnos sean activos con su aprendizaje deben ser responsables con sus tareas, interactuar de manera efectiva con sus asesores y autoevaluar su desempeño. Investigaciones como la de Braxton, Jones, Hirschy y Hartley (2008) han mostrado la importancia del aprendizaje activo en la retención de información que obtienen estudiantes universitarios de primer año. Chickering y Gamson (1987) formularon siete principios de buenas prácticas para la licenciatura y uno de ellos es fomentar el aprendizaje activo. Considerando el potencial del Aprendizaje 2.0 y la relevancia que tiene el aprendizaje activo en el rendimiento académico, la inclusión de estrategias instruccionales vinculadas a la Web 2.o debería ser imprescindible en la educación universitaria actual.

Bonwell y Eison (1991) han encontrado algunas características asociadas con el aprendizaje activo, como: a) los estudiantes se involucran en algo más que sólo escuchar; b) el énfasis está centrado en el desarrollo de habilidades; c) los estudiantes se involucran en pensamiento de orden superior (análisis, síntesis, evaluación); d) los estudiantes se comprometen con actividades diversas tales como la lectura, la escritura y la discusión; y e) hay un mayor énfasis en la exploración de actitudes y valores de los estudiantes. 
Los LMS ofrecen múltiples recursos que facilitan el Aprendizaje 2.o y el aprendizaje activo. De igual modo, informan a los estudiantes sobre los objetivos didácticos, competencias a formar y contenidos de cada sección. Cuentan con herramientas de comunicación y colaboración que permiten socializar el conocimiento como los foros de discusión, los wikis y el chat, herramientas de gestión de contenidos que facilitan a los estudiantes los materiales, herramientas de gestión de grupos que promueven el aprendizaje cooperativo y herramientas de seguimiento y evaluación (Fernández \& Rivero, 2014).

El efecto de las estrategias instruccionales alojadas en herramientas tecnológicas para mejorar el aprendizaje es motivo de interés para muchos investigadores (Gabrie1le, 2003; Lumpkin, Achen \& Dodd, 2015). Sin embargo, a pesar de los valiosos esfuerzos de estudiosos de la educación, persisten algunas resistencias para enseñarlas y éstas siguen afectando a docentes y alumnos. Monereo, Castelló, Clariana, Palma y Pérez (1994) señalan las siguientes resistencias: a) la formación en estrategias de aprendizaje es improductiva debido a los conocimientos previos de los docentes para manejarlas en el aula, especialmente cuando implican una dificultad superior a los contenidos de la materia; b) algunas estrategias solamente aplican para cierto tipo de alumnos; y c) formar en estrategias de aprendizaje constituye un lujo porque hay asuntos más relevantes como los contenidos de cada materia.

Las estrategias permiten la toma de decisiones adecuadas en el proceso de aprendizaje (Beltrán \& Bueno, 1997) y esa toma de decisiones (conscientes e intencionales) permite seleccionar los conocimientos necesarios para cumplir con un objetivo (Monereo et al., 1994). Otro punto a considerar es el valor de las estrategias para facilitar la activación de la mente de los estudiantes (Schneider \& Stern, 2010).

Para desarrollar las habilidades que se requieren en cada una de las dimensiones competenciales de la alfabetización digital es necesario considerar, en primer lugar, las escasas habilidades cognoscitivas y conocimientos estructurales con los que ingresan los estudiantes al nivel universitario. Backhoff y Tirado (1993) realizaron una investigación en la Universidad Autónoma de Baja California (UABC), donde utilizaron como instrumento el Examen de Habilidades y Conocimientos Básicos (EXHCOBA). Evaluaron los conceptos estructurales y destrezas cognoscitivas y distribuyeron los reactivos en tres secciones: habilidades básicas (nivel primaria), conocimientos básicos (nivel secundaria) y conocimientos básicos para especialidad (nivel bachillerato).

Los autores explican que los resultados de todas las secciones se pueden agrupar en un rango de aciertos que oscilan desde $20 \%$ hasta $57 \%$. Tales resultados re- 
probatorios reflejan el bajo nivel académico de egreso del bachillerato. La información proporcionada por el ЕхнсовA se puede contrastar con datos más recientes de los niveles de rendimiento educativo de pruebas como el Programa para la Evaluación Internacional de Alumnos (PISA, por sus siglas en inglés) que se aplica a estudiantes de 15 años. PISA 2015 reflejó que el "desempeño de México se encuentra por debajo del promedio Organización para la Cooperación y el Desarrollo Económicos (OCDE) en ciencias (416 puntos), lectura (423 puntos) y matemáticas (408 puntos)" (OCDE, 2016). Los datos mencionados son motivo de gran preocupación para los docentes de nivel universitario, debido a que los conocimientos previos que poseen los egresados de la Educación Media Superior son insuficientes para los contenidos que deben asimilarse y comprenderse en los diferentes semestres de las licenciaturas. Sería deseable mejorar las habilidades y conocimientos que se transmiten en la Educación Básica para que los estudiantes lleguen mejor preparados a la universidad.

En segundo lugar, con el aumento de la oferta educativa en línea de universidades públicas y privadas, la apropiación de las tecnologías de la información y la comunicación (TIC) se convierte en un tema primordial para el rendimiento académico. Parte del aprendizaje obtenido depende de la alfabetización digital. Según la Organización de las Naciones Unidas para la Educación, la Ciencia y la Cultura (UNESCO, 2011), la alfabetización digital es un conjunto de habilidades del usuario que le permiten una participación activa en una sociedad donde los servicios y la oferta cultural se basan en las computadoras y se distribuyen en Internet. A pesar del papel que juega la alfabetización digital en la educación, se ha encontrado que los estudiantes usan las TIC, pero no se apropian de ellas pensando en su potencial productivo, sino que se limitan a los usos básicos y recreativos (Berrío-Zapata \& Rojas, 2014).

Monereo (2009) destaca el hecho de que, aunque los nativos digitales tengan acceso a la tecnología, no hay garantía de que tengan "los bits bien puestos". Menciona algunas deficiencias para actividades de aprendizaje como la búsqueda y selección de información, problemas para evaluar la confiabilidad de la información y trabajar en equipo, compulsión para hacer varias cosas a la vez y dificultades para la administración del tiempo.

Usar la tecnología para el aprendizaje aporta diferentes beneficios que contribuyen a la alfabetización digital: 1) modulan la estructura cognitiva; 2) modifican la capacidad de concentración; 3) transforman la autorregulación del aprendizaje y 4) modifican la forma de relacionarse (Cabero \& Barroso, 2015). 
La posición del alumno ante el aprendizaje mediado por tecnología debe transformarse hacia una nueva manera de aprender y al uso de nuevos métodos y técnicas (Martín-Laborda, 2005), y para ser eficaces y eficientes en esa transformación, los estudiantes universitarios deben ser competentes para leer y comprender lo que leen; competentes para escribir con coherencia y cohesión; competentes para autorregular su aprendizaje y competentes para aprovechar las herramientas Web 2.o para su aprendizaje.

Fonseca, Medellín y Vázquez (2014) refieren que la combinación de tecnología Web 2.0 y estrategias didácticas adaptadas al tipo de contenido, son de gran utilidad para el aprendizaje a través de actividades dinámicas. Dicha mancuerna, Web 2.o-estrategias didácticas, permitirá enriquecer el aprendizaje en la Educación Superior en la medida en que docentes y directivos de instituciones educativas se comprometan a promover y fomentar su uso y apropiación.

Por la relevancia del uso de estrategias combinado con la tecnología Web 2.0, el principal objetivo de este estudio fue explorar la autopercepción sobre habilidades académicas que se requieren para el aprendizaje en ambientes virtuales que poseen estudiantes universitarios matriculados en cursos mediados por tecnología.

\section{Método}

Se utilizó un diseño metodológico de carácter descriptivo cuantitativo. Se diseñó una encuesta para explorar la autopercepción sobre las habilidades para la comprensión lectora, comunicación escrita, aprendizaje autónomo y uso de herramientas Web 2.o que poseen estudiantes universitarios matriculados en cursos mediados por tecnología. Dichas habilidades se eligieron por tener una relación directa con las dimensiones de la alfabetización digital y con la capacidad de aprender de manera autónoma.

Para diseñar la encuesta se revisaron las pruebas disponibles sobre estrategias de aprendizaje para identificar las principales habilidades involucradas en leer, escribir, aprender en solitario y usar herramientas Web 2.0 para propósitos de estudio. Del análisis de varios instrumentos formados por una gran cantidad de reactivos, se eligieron las habilidades más mencionadas para construir un instrumento con una extensión que no dificultara el proceso de respuesta de los estudiantes. El instrumento abreviado de 33 reactivos explora cuatro aspectos fundamentales para aprender 
en ambientes virtuales y desarrollar las dimensiones de la alfabetización digital en la universidad: comprensión lectora, comunicación escrita, aprendizaje autónomo y uso de tecnología, que aportarían información para responder a la pregunta de investigación planteada.

Se realizó una prueba piloto para validar el instrumento donde participaron 120 estudiantes de carreras de Ciencias Sociales de una universidad pública. Se obtuvo un Alfa de Cronbach de .88, con lo cual se asegura que el instrumento es fiable.

El cuestionario se compone de seis secciones:

1. Información demográfica. En esta sección se incluyeron preguntas sobre la carrera cursada, tipo de universidad, sexo y edad.

2. Plataformas educativas. Enfocada a identificar si cursa o no una materia en plataforma educativa, nombre de la plataforma, actividades que realiza y dispositivo utilizado.

Para las secciones III, IV y V se usó una Escala de Likert con cinco niveles desde nunca hasta siempre.

3. Comprensión lectora. Consiste en ocho preguntas para explorar las percepciones sobre el uso de estrategias indispensables para la comprensión de instrucciones para realizar actividades en línea, la disposición para aclarar dudas con los asesores y el interés por consultar textos para comprender temas y enriquecer sus documentos académicos.

4. Comunicación escrita. Contiene ocho preguntas para explorar las percepciones sobre el uso de estrategias para comunicarse por escrito, tales como el uso de organizadores gráficos, redacción de borradores, búsqueda de fuentes de información complementarias y uso del procesador de texto.

5. Aprendizaje autónomo. Incluye nueve preguntas para identificar las percepciones de los estudiantes para aprender de forma autónoma como la planeación, selección de puntos más importantes, comportamiento ante dudas, aclaración de dudas de forma presencial o usando herramientas tecnológicas y evaluación del proceso de aprendizaje.

6. Herramientas Web 2.o. A través de ocho preguntas se exploraron las percepciones sobre los conocimientos de los estudiantes sobre diferentes herramientas Web 2.o. Se usó una Escala de Likert con cinco niveles, de deficiente a excelente.

Se eligió un muestreo por cuotas en donde se seleccionó a estudiantes universitarios de la licenciatura de Derecho de la Universidad Autónoma de Querétaro, pertene- 
cientes a la generación conocida como nativos digitales, que utilizan un LMS (Moodle) como apoyo en sus materias. Participaron 120 estudiantes, de los cuales 67 eran mujeres y 53, hombres. La edad promedio fue de 21 años. El estudio se realizó en el semestre comprendido de enero a junio de 2016.

\section{Análisis y resultados}

El principal objetivo de este estudio fue explorar la percepción que tienen los estudiantes matriculados en cursos mediados por tecnología sobre habilidades relacionadas con la alfabetización digital y el nivel de conocimientos que poseen sobre las herramientas Web 2.o.

En primer término, se explican los resultados demográficos y posteriormente se presentan los resultados sobre las percepciones que tienen sobre comprensión lectora, comunicación escrita, aprendizaje autónomo y uso de las herramientas Web 2.o.

\subsection{Resultados demográficos}

Para la investigación, se eligió una universidad pública que utiliza el LMS Moodle. Los participantes mencionaron que el dispositivo que más utilizan para ingresar al campus virtual con el fin de realizar consultas de contenidos y materiales es la laptop, superando a las computadoras personales (PC) por un margen considerable (57\% vs. $30 \%$, respectivamente), lo cual puede deberse a la portabilidad de la primera. Es importante destacar que 33\% de los estudiantes mencionó que usa más de un dispositivo.

Las actividades que realizan dentro del campus virtual son: participación en foros, participación en wikis, lectura de materiales educativos básicos, envío de correos electrónicos, revisión y análisis de tareas por entregar, consulta de los contenidos de la asignatura, lectura de materiales educativos complementarios, uso de chat y consulta de información general (temarios, objetivos, competencias). En la Figura 1 se presenta el desglose por actividad. 


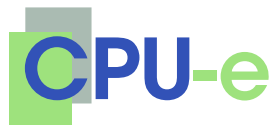

Autopercepción de habilidades de aprendizaje en ambientes virtuales

Ana María Orozco Santa María y Ma. Teresa García Ramírez

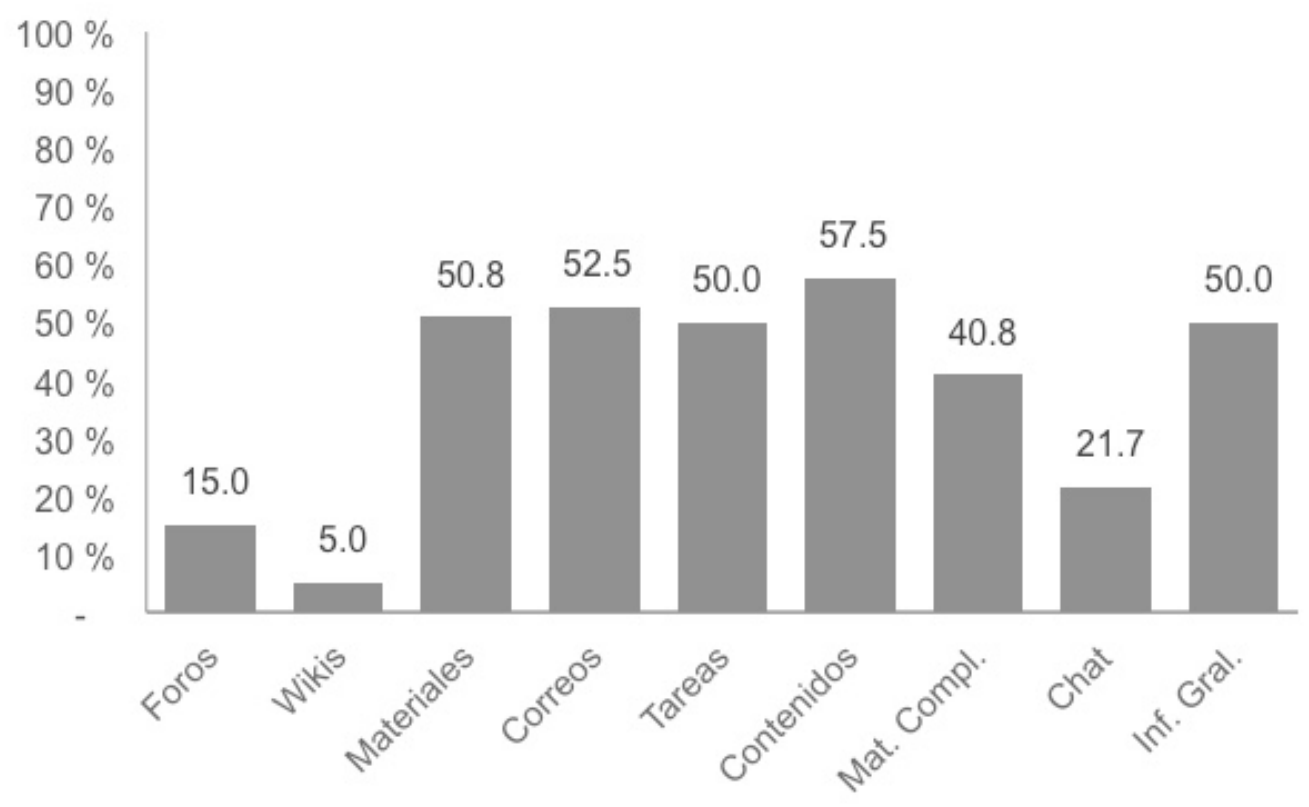

Figura 1. Uso del campus virtual (cantidad de alumnos)

Fuente: Elaboración propia.

Como se puede observar, la mayoría de las actividades disponibles dentro de un LMS son usadas por más de $42 \%$ de los estudiantes que participaron en el estudio. Destaca el hecho de que los porcentajes más bajos de uso lo tienen la lectura de materiales complementarios y el uso del chat, foros $y$ wikis.

\subsection{Comprensión lectora}

Fainholc (2004) menciona que existe una necesidad de alfabetización en las TIC para el desarrollo de competencias de interacción y nuevas prácticas de lectura. Ante tal afirmación de una docente que se ha dedicado a la investigación en tecnología educativa, se pensó en indagar si los estudiantes comprenden las instrucciones; solamente $30 \%$ declaró que siempre lo hace, y 52\% lo hace "muchas veces" (Figura 2). 


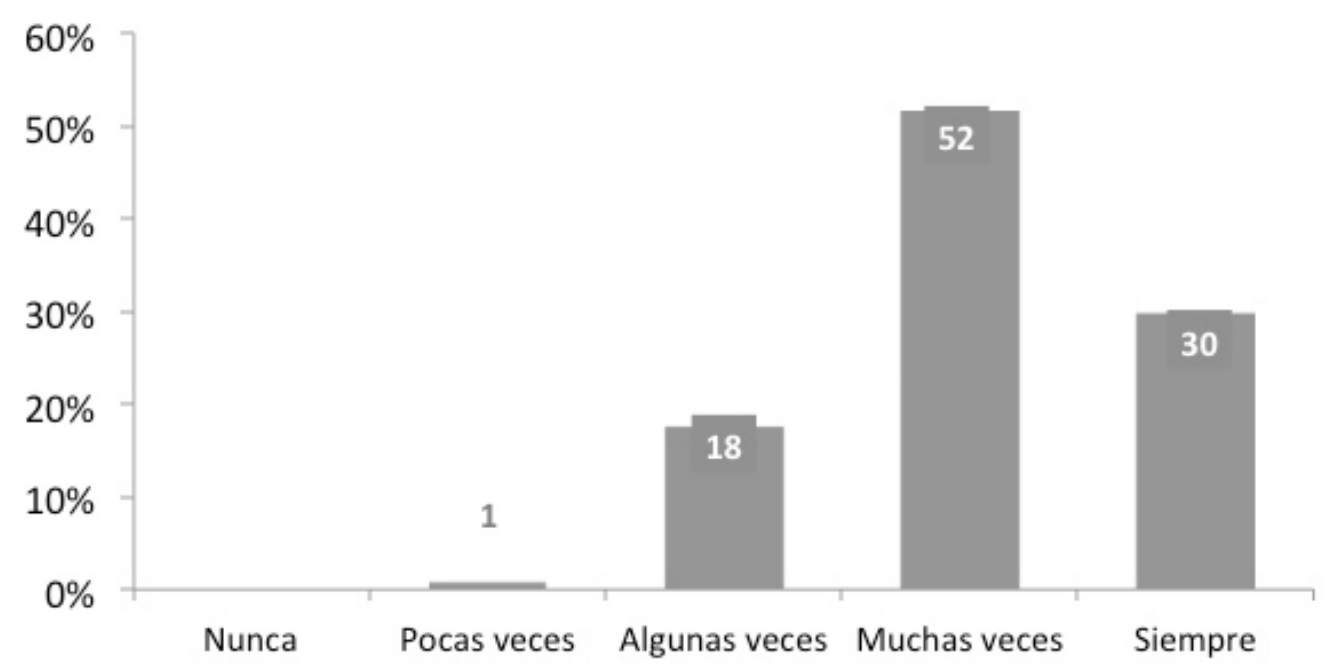

Figura 2. Comprensión de las instrucciones

Fuente: Elaboración propia.

Esto podría significar que, a pesar de que la mayoría de los estudiantes dicen sí comprender las instrucciones, hay un porcentaje que no cumplirá en su totalidad con lo solicitado por no entender lo que debe hacer. Los datos reflejan la situación que se detecta de manera empírica cuando los estudiantes entregan trabajos que no cumplen con las especificaciones solicitadas. No comprender las instrucciones de una actividad impacta negativamente en el aprendizaje de los contenidos y en la motivación para aprender usando un LMS. Por ejemplo, estudios previos (Arrieta \& Meza, 2005; Massone \& González, 2005) muestran que los estudiantes universitarios presentan dificultades para entender lo que leen y tienen problemas para seguir instrucciones orales y por escrito.

En los reactivos sobre comprensión lectora, también se incluyó una pregunta sobre una habilidad cognitiva fundamental para el aprendizaje significativo: relacionar el material nuevo con conocimientos previos. El estudio mostró que $27 \%$ de los encuestados utiliza esta estrategia siempre y $41 \%$, muchas veces (Figura 3). Asimismo, los resultados obtenidos reflejan que hay estudiantes que la utilizan pocas veces o nunca. Tal situación podría significar que no saben cómo establecer dichas relaciones o bien, que no poseen suficientes conocimientos previos para anclarlos con el nuevo aprendizaje. Conocer esta información es de utilidad para los maestros porque les 
indica qué áreas necesitan reforzar en sus estudiantes. Al promover la relación de los nuevos conocimientos con los previos, podrá ayudarse al estudiante a producir un aprendizaje más duradero.

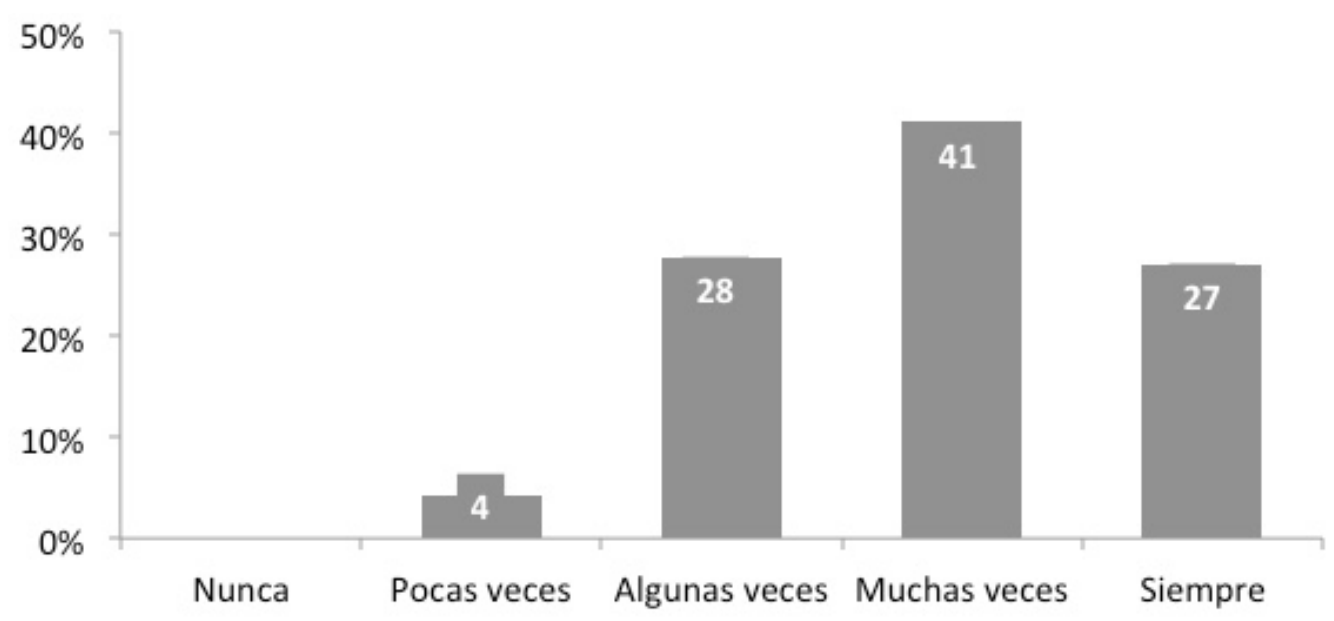

Figura 3. Relaciono material nuevo con conocimientos previos

Fuente: Elaboración propia.

\subsection{Comunicación escrita}

Como se mencionó anteriormente, la comprensión es fundamental para el aprendizaje significativo, por lo que se indagó si los estudiantes utilizan organizadores gráficos para ordenar sus ideas. La web ofrece diferentes herramientas gratuitas para crear mapas conceptuales como CmapTools o Mindomo, y para crear mapas mentales como Wisemapping o Mindmeinster. A pesar de todas las herramientas disponibles, solamente $4 \%$ declaró usarlas siempre, y $23 \%$ dijo no usarlas nunca (Figura 4). Los mapas mentales y los mapas conceptuales constituyen una estrategia muy útil, ya que permiten esquematizar y organizar información que hará que los estudiantes la procesen con mayor facilidad. Al estudiar cualquier tema, es "fundamental la interacción significativa del alumno con la tarea, en lugar de una captación meramente superficial y repetitiva” (Ontoria, Ballesteros, Cuevas, Giraldo, Martín, Molina, Rodríguez \& Vélez, 2000, p. 27). Una vez que el estudiante comprende un tema, estará en posibilidades de escribir sobre el mismo con coherencia y cohesión. 


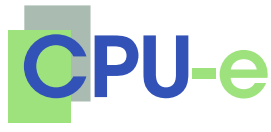

Autopercepción de habilidades de

aprendizaje en ambientes virtuales

Ana María Orozco Santa María y Ma. Teresa García Ramírez

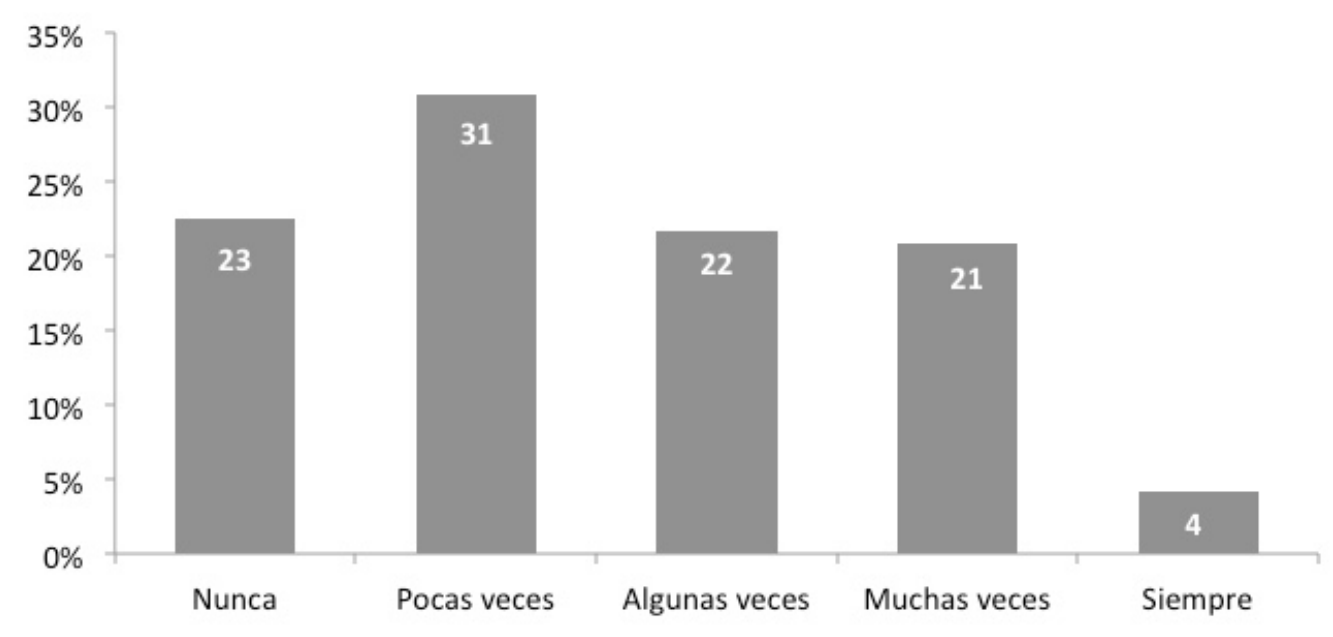

Figura 4. Uso de organizadores gráficos para ordenar ideas.

Fuente: Elaboración propia.

La tecnología ofrece muchas herramientas de apoyo para actividades relacionadas con la comunicación escrita, por lo que se exploró el nivel de uso que dan los estudiantes al procesador de textos para verificar gramática y ortografía; 42\% mencionó que siempre lo usa, pero hay estudiantes que lo usan poco o nunca (Figura 5). La gran cantidad de estudiantes que recurre a este tipo de funciones podría explicarse por lo extendido del uso de procesadores de texto comerciales que permiten tales ayudas con ortografía y gramática. Sin embargo, los datos sugieren que algunos alumnos no usan o no saben usar las funcionalidades tipográficas, idiomáticas u organizativas de esta aplicación informática.

Actualmente, los procesadores de textos comerciales tienen aplicaciones que marcan los errores con subrayados en color. Para enfrentar una actividad de aprendizaje que produzca documentos con alta calidad académica, sería deseable que los estudiantes usaran las herramientas que están a su alcance en los dispositivos que utilizan cotidianamente. 


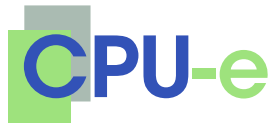

Autopercepción de habilidades de

aprendizaje en ambientes virtuales

Ana María Orozco Santa María y Ma. Teresa García Ramírez

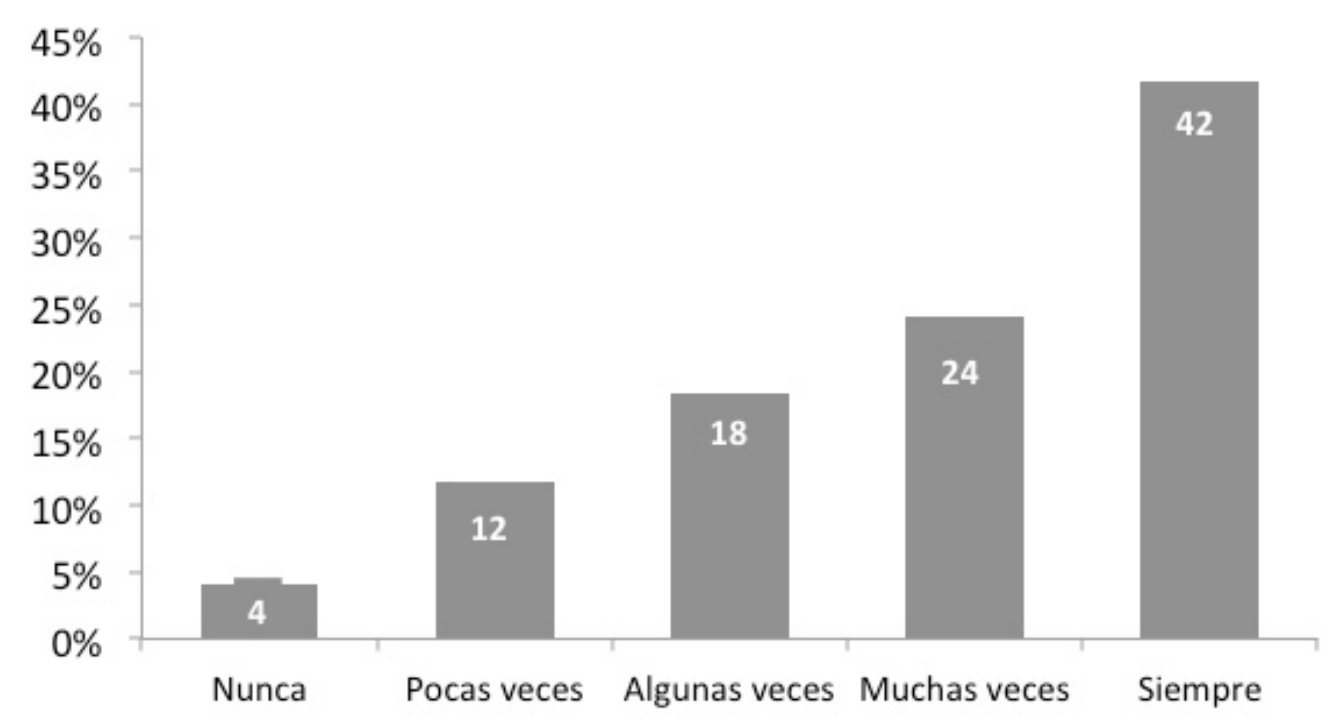

Figura 5. Uso el procesador de textos para verificar gramática y ortografía

Fuente: Elaboración propia.

La apropiación tecnológica implica interés y motivación, y la investigación de BerríoZapata y Rojas (2014) realizada en Bogotá identificó que los estudiantes usan las TIC, pero "no se apropian de sus posibilidades productivas más allá de los usos básicos o recreativos" (p. 140).

Los organizadores gráficos y el procesador de textos son recursos que proveen de ventajas diversas a los estudiantes y su apropiación no se ha logrado todavía. Se debe mostrar a docentes y estudiantes los beneficios de usar la tecnología vinculada con diferentes estrategias para mejorar la calidad del aprendizaje.

\subsection{Aprendizaje autónomo}

Los resultados de investigaciones sobre el rol del estudiante a distancia establecen que deben ser responsables, realizar consultas y autoevaluar su desempeño (Isman, Altinay \& Altinay, 2004). Considerando que la autonomía es fundamental para obtener buenos resultados en esta modalidad educativa, se exploraron algunos aspectos como la planificación para el estudio, selección del material más relevante, aclaración 
de dudas y autoevaluación. El estudio expuso que la mayoría de los estudiantes está entre "algunas veces" y "nunca" en esta categoría (Figura 6), lo que significa que hay una cantidad importante de estudiantes que no planifica el estudio. La falta de planeación puede impactar en entregas extemporáneas de actividades y tareas que nunca son entregadas, lo cual afecta el aprendizaje y la calificación de los estudiantes.

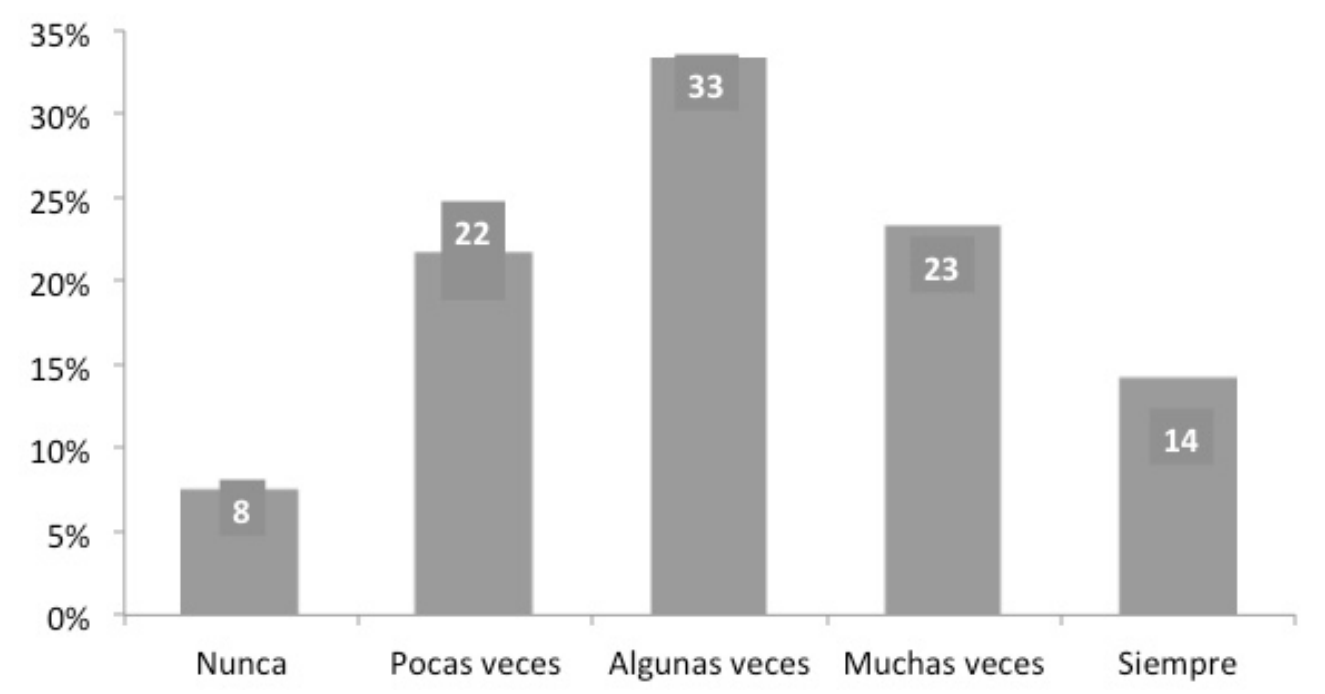

Figura 6. Planifico el tiempo para cada asignatura y actividad de aprendizaje

Fuente: Elaboración propia.

La autonomía en el estudio es una habilidad fundamental en los cursos mediados por tecnología y los resultados reafirmaron que los docentes deben fomentar la autorregulación. Promover la autonomía en el estudio permitirá que los estudiantes que no acostumbran a planificar el tiempo, sean conscientes de los beneficios de hacerlo. De igual manera, los alumnos que lo hacen pocas o algunas veces deben reconocer las ventajas de administrar su tiempo.

La comunicación con el profesor debe ser constante para aclarar dudas y compartir comentarios sobre los contenidos y materiales; sin embargo, los resultados del estudio muestran que solamente $5 \%$ siempre aclara sus dudas con el profesor utilizando los medios electrónicos a su alcance, y más de 50\% está entre "nunca" y "pocas 
veces", lo que significa que la comunicación con el profesor es un problema para los estudiantes usuarios de LMS (Figura 7).

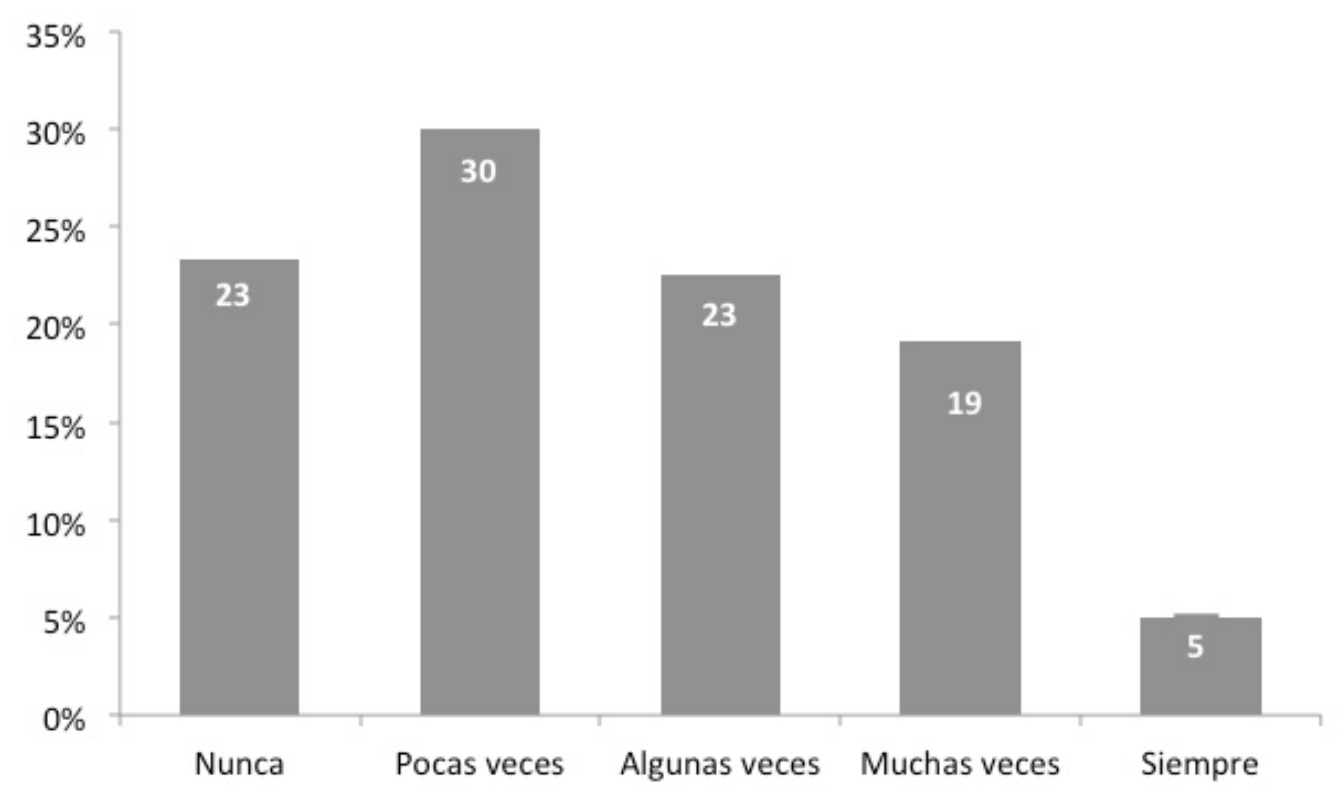

Figura 7. Aclaro dudas con el profesor usando herramientas tecnológicas

Fuente: Elaboración propia.

Como lo señala Salinas (2008), dentro de los entornos virtuales el rol del alumno debe ser más activo y ello implica la planificación de las actividades, la comprensión e interpretación de la información, la búsqueda de información para enriquecer el conocimiento y una comunicación efectiva y constante con los profesores en todo momento.

\subsection{Herramientas Web 2.o}

Area (2010) señala que "precisamos ser más competentes que en décadas anteriores para poder emplear y apropiarnos de la información y la tecnología digital” (p.3), por lo que conocer la percepción que tienen los estudiantes sobre el uso de las herramientas Web 2.0 es indispensable para establecer las acciones necesarias para promover $y$ difundir sus usos profesionales. 
Los foros de discusión y los wikis son de las herramientas más usadas en Educación Superior debido a que favorecen el desarrollo de diferentes habilidades, como la argumentación, la comunicación escrita y el trabajo colaborativo. A pesar de que son las herramientas que se incorporan con mayor frecuencia en los cursos a distancia, el estudio muestra que sólo $7 \%$ declaró que siempre utiliza los foros y la cifra en el caso de los wikis, se reduce a $1 \%$.

Un dato interesante es el relativo a los archivos en la nube; $29 \%$ respondió que siempre usa la nube para guardar información. De todas las herramientas consideradas en el estudio, ésta es la que obtuvo el porcentaje más alto en la columna "siempre" y puede deberse a la difusión que han tenido los servicios de almacenamiento en la nube, ya que son muy cómodos y evitan el uso de dispositivos que pueden llegar a perderse o dañarse. Con los datos obtenidos, se corrobora lo que otras investigaciones han descubierto sobre el nivel superficial de apropiación tecnológica en estudiantes universitarios (Berrío-Zapata \& Rojas, 2014; Lemus, Garza \& Caloca, 2011).

Con el propósito de identificar las diferencias existentes entre las habilidades académicas estudiadas, se realizó un análisis ANOva de medidas repetidas. Los datos utilizados se muestran en la Tabla 1.

Tabla 1. Estadística descriptiva de habilidades académicas

\begin{tabular}{ccccc}
\hline & $\begin{array}{c}\text { Comprensión } \\
\text { lectora }\end{array}$ & $\begin{array}{c}\text { Comunicación } \\
\text { escrita }\end{array}$ & $\begin{array}{c}\text { Aprendizaje } \\
\text { autónomo }\end{array}$ & $\begin{array}{c}\text { Herramientas } \\
\text { Web 2.0 }\end{array}$ \\
\hline Media & 75.4 & 71.1 & 65.9 & 56.6 \\
\hline $\begin{array}{c}\text { Desviación } \\
\text { estándar }\end{array}$ & 10.7 & 11.4 & 12.8 & 18.8 \\
\hline $\mathrm{N}$ & 120 & 120 & 120 & 120 \\
\hline
\end{tabular}

Fuente: Elaboración propia.

La Figura 8 muestra el nivel de habilidades académicas por competencia. 


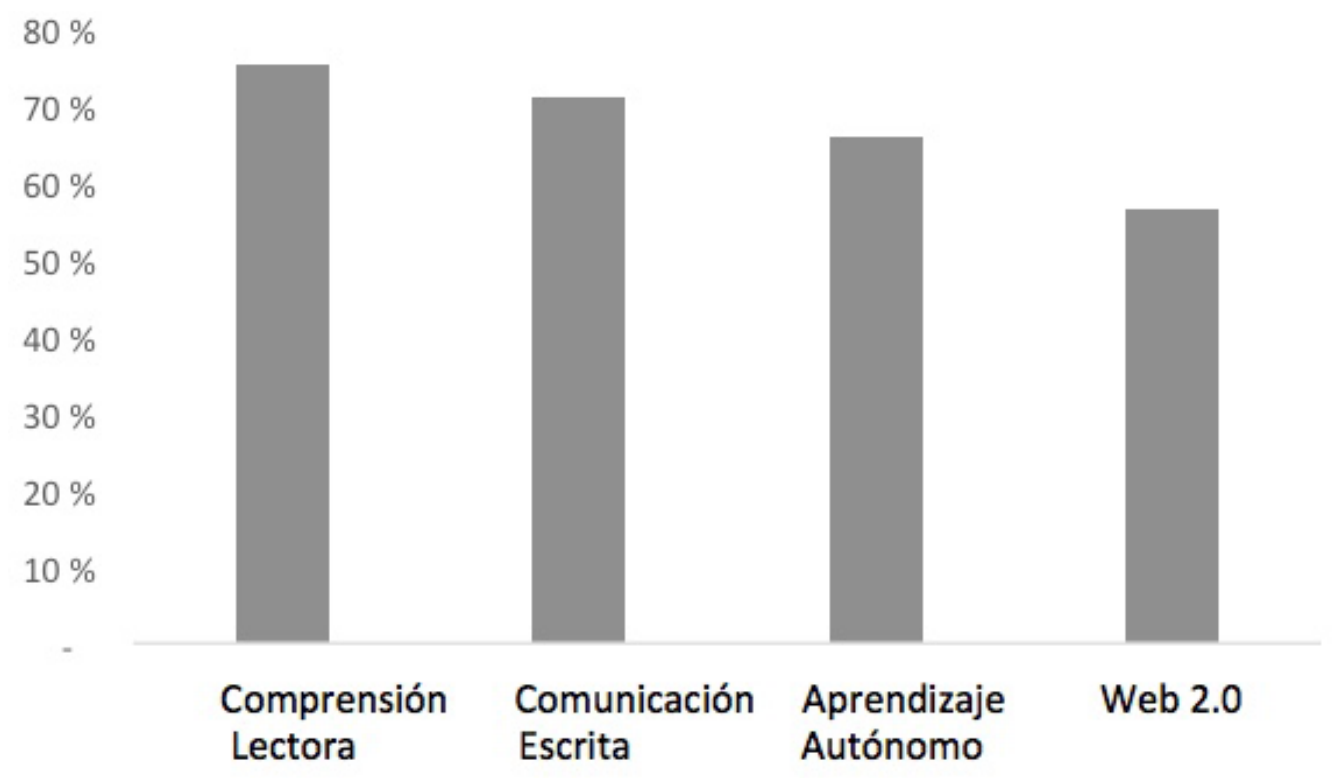

Figura 8. Nivel de habilidades académicas por competencia

Fuente: Elaboración propia.

La ANOvA reporta que hay diferencias significativas entre las dimensiones evaluadas, $\mathrm{F}(3,119)=69.2, \mathrm{p}<.001$. También se realizó una prueba post hoc de Bonferroni, la cual reveló que hay diferencias significativas $(\mathrm{p}<.001)$ entre todas las dimensiones estudiadas.

Como lo muestra el análisis, las competencias con mejores resultados son comprensión lectora y comunicación escrita. A pesar de ello, se hace evidente la necesidad de trabajar en dichas habilidades académicas para lograr niveles más altos. Por ejemplo, para mejorar la comprensión lectora en la universidad, Carlino (2005) sugiere que es indispensable "leer bibliografía auténtica de una disciplina para poder analizar en profundidad las corrientes teóricas, apreciar la complejidad de sus razonamientos y examinar las bases empíricas en las que se apoyan” (p. 73). Propone como ayuda para los estudiantes una serie de preguntas que sirvan de guía para enfocar la lectura. Sobre los problemas relativos a la escritura, Carlino (2005) explica que no sólo se deben a una mala formación en los niveles educativos previos. Se trata también de "nuevas formas discursivas que desafían a todos los principiantes y que, para muchos de ellos, 
suelen convertirse en barreras insalvables si no cuentan con docentes que los ayuden a atravesarlas" (p. 23). Por tanto, sugiere que los docentes de cualquier materia se ocupen de la escritura, ya que, "hacerse cargo de enseñar a leer y a escribir en la universidad es ayudar a los alumnos a aprender" (p. 24)

Las habilidades académicas que presentan los niveles más bajos son aprendizaje autónomo y herramientas Web 2.o. Dichos datos se relacionan con lo expresado por Monereo (2009) acerca de las dificultades que presentan los nativos digitales para gestionar sus tiempos y que, aunque estén rodeados de pantallas, no es garantía de que tengan los "bits bien puestos".

Tal como lo señala Monereo (2009), la alfabetización digital es una segunda alfabetización que implica reorganizar competencias ya adquiridas.

\section{Conclusiones}

En este estudio se exploró lo que perciben los estudiantes universitarios matriculados en cursos mediados por tecnología sobre las habilidades requeridas para ser individuos digitalmente alfabetizados.

Las dos habilidades en las que los alumnos se perciben mejor fueron comprensión lectora y comunicación escrita. Ambas influyen directamente en la capacidad para incorporar nuevos conocimientos y comunicarlos de manera efectiva, por lo que son fundamentales para la dimensión cognitiva de la alfabetización digital.

El estudio mostró que se sienten menos cómodos cuando se requiere más actividad o interacción de su parte, por ejemplo, para elaborar organizadores gráficos o comunicarse con su tutor para solución de dudas. Un número muy limitado de estudiantes reconoció usar organizadores gráficos, y su utilización ayuda a la comprensión, remembranza y aprendizaje.

Otro aspecto que merece especial atención es la comunicación usando herramientas tecnológicas, ya que todavía hay muchos estudiantes que no acuden a su profesor para aclarar dudas teniendo la posibilidad de hacerlo.

Elaborar organizadores gráficos y comunicarse con los maestros usando la tecnología son dos actividades para las que no han sido formados dentro de la educación tradicional. Fomentar el uso de herramientas para crear mapas mentales y conceptuales permitirá contribuir al desarrollo de habilidades para la lectura, análisis, comprensión y recreación de información. Asimismo, es recomendable incluir estrategias 
instruccionales para motivar y guiar a los estudiantes para que se comuniquen eficazmente usando la tecnología.

Muchas instituciones están apostando a la educación a distancia y parece ser que los estudiantes no están aún listos para enfrentar los retos de un aprendizaje en solitario mediado por la tecnología. Tal escenario es un indicador de que los docentes deben usar diferentes herramientas para fomentar la interacción y la autonomía en el aprendizaje. Por su parte, los estudiantes deben ver a la tecnología como una aliada en su aprendizaje y no solamente como un dispositivo para divertirse y comunicarse. Asimismo, deberán tener un control más riguroso de su tiempo para administrar sus actividades y obtener mejores resultados.

Es recomendable que los docentes encargados del diseño de cursos en línea consideren, además de los contenidos, las diferentes dimensiones de la alfabetización digital como una guía imprescindible para desarrollar habilidades instrumentales, cognitivas, sociocomunicacionales, axiológicas y emocionales.

Para cumplir con el compromiso de formar estudiantes digitalmente alfabetizados, el aprendizaje activo constituye una estrategia esencial para promover la participación y la reflexión a través de actividades que pueden trabajarse usando diferentes herramientas tecnológicas.

\section{Lista de referencias}

Amaya, J., \& Prado, E. (2002). Estrategias de aprendizaje para universitarios: un enfoque constructivista. México: Trillas.

Area, M. (julio, 2010). ¿Por qué formar en competencias informacionales y digitales en la educación superior? Revista de Universidad y Sociedad del Conocimiento, 7(2). Recuperado de http://www.redalyc.org/articulo.oa?id=78016225012

Area, M., Moreira, A., \& Vidal, F. (8, noviembre, 2012). Alfabetización digital y competencias informacionales [Página web]. Recuperado de https://www.fundaciontelefonica.com/arte_cultura/publicaciones-listado/pagina-item-publicaciones/itempubli/161/

Arrieta, B., \& Meza, R. (2005). La comprensión lectora y la redacción en estudiantes universitarios. Revista Iberoamericana de Educación, 35(2). Recuperado de http://rieoei.org/didactica8.htm

Backhoff, E., \& Tirado, F. (octubre-diciembre, 1993). Habilidades y conocimientos 
básicos del estudiante universitario: hacia los estándares nacionales. Revista de la Educación Superior, 22(88). Recuperado de http://publicaciones.anuies. $\mathrm{mx} /$ revista/88/1/4/es/habilidades-y-conocimientos-basicos-del-estudianteuniversitario

Bartolomé, A., \& Grané, M. (octubre, 2004). Educación y tecnologías: de lo excepcional a lo cotidiano. Aula de Innovación Educativa, 135. Recuperado de https:// www.researchgate.net/publication/39209998_Educacion_y_Tecnologia de lo excepcional a lo cotidiano

Beltrán, J., \& Bueno, J. (Eds.). (1997). Psicología de la educación. Barcelona: Alfaomega. Berrío-Zapata, C., \& Rojas, H. (2014). La brecha digital universitaria: La apropiación de las TiC en estudiantes de Educación Superior de Bogotá (Colombia). Revista Científica de Educomunicación, 43(22). Recuperado de https://www. revistacomunicar.com/index.php? contenido $=$ detalles\&numero $=43 \&$ articu $\underline{\mathrm{lo}=43-2014-13}$

Bonwell, C., \& Eison, J. (1991). Active learning: creating excitement in the classroom. (Report No. 1). Washington, D.C.: School of Education and Human Development-The George Washington University. Recuperado de http://files.eric. ed.gov/fulltext/ED 336049.pdf

Braxton, J. M., Jones, W. A., Hirschy, A. S., \& Hartley, H. V. (2008). The role of active learning in college student persistence. New directions for teaching and learning, 115, 71-83. Recuperado de https://eric.ed.gov/?id=EJ811655

Cabero, J., \& Barroso, J. (Coords.). (2015). Nuevos retos en tecnología educativa. Madrid: Síntesis.

Carlino, P. (2005). Escribir, leer y aprender en la universidad: una introducción a la alfabetización académica. Buenos Aires: Fondo de Cultura Económica de Argentina.

Chickering, A., \& Gamson, Z. (1987). Seven principles for good practice. Recuperado de http://www.lonestar.edu/multimedia/SevenPrinciples.pdf

Dewey, J. (1989). Cómo pensamos: Nueva exposición de la relación entre pensamiento reflexivo y proceso educativo. Barcelona: Paidós Ibérica.

Díaz-Barriga, F., \& Hernández, G. (2002). Estrategias docentes para un aprendizaje significativo ( $2^{\mathrm{a}} \mathrm{ed}$.). México: McGraw-Hill Interamericana.

Estéfano, R. (julio-diciembre, 2013). Conocimiento y aplicación de estrategias de aprendizaje por profesores de Educación superior a distancia. Zona Próxima, 19, 21-38. Recuperado de http://www.redalyc.org/pdf/853/85329192003.pdf 
Fainholc, B. (2004). Lectura crítica en Internet: análisis y utilización de los recursos tecnológicos en Educación. Argentina: Homo Sapiens.

Felder, R., \& Brent, R. (2005). Understanding student differences. Journal of Engineering Education, 94(1), 57-62. Recuperado de http://www4.ncsu.edu/unity/ lockers/users/f/felder/public/Papers/Understanding Differences.pdf

Fernández, A., \& Rivero, M. (2014). Las plataformas de aprendizajes, una alternativa a tener en cuenta en el proceso de enseñanza aprendizaje. Revista Cubana de Informática Médica, 6(2), 207-221. Recuperado de http://www.rcim.sld.cu/revista_29/articulo htm/plataformasaprendizajes.htm

Fonseca, L. B., Medellín, L. A., \& Vázquez, J. L. (2014). El uso de las herramientas Web 2.0 como estrategias didácticas en el proceso de enseñanza-aprendizaje de jóvenes universitarios. Paakat, Revista de tecnología y sociedad, 7. Recuperado de http://www.udgvirtual.udg.mx/paakat/index.php/paakat/article/ view $/ 227 / 340$

Gabrielle, D. M. (2003). The Effects of Technology-Mediated Instructional Strategies on Motivation, Performance, and Self-Directed Learning. En D. Lassner \& C. McNaught (Eds.), Proceedings of EdMedia: World Conference on Educational Media and Technology 2003 (pp. 2568-2575). Estados Unidos: Universidad Estatal de Florida.

Hernández, G. (1998). Paradigmas en psicología de la educación. México: Paidós.

Hernández, G., \& Díaz Barriga, F. (enero-junio, 2013). Una mirada psicoeducativa al aprendizaje: qué sabemos y hacia dónde vamos. Sinéctica, 40. Recuperado de https://sinectica.iteso.mx/index.php/sinecticA/article/view/44

Hernández, D., Ramírez, A., \& Cassany, D. (enero, 2014). Categorizando a los usuarios de sistemas digitales. Pixel-Bit. Revista de medios y educación, 44, 113-126. Recuperado de http://acdc.sav.us.es/ojs/index.php/pixelbit/article/view/103/7

Isman, A., Altinay, Z., \& Altinay, F. (octubre, 2004). Roles of the Students and Teachers in Distance Education. Turkish Online Journal of Distance Education, 5(4). Recuperado de http://tojde.anadolu.edu.tr/yonetim/icerik/makaleler/162published.pdf

Lemus, M. C., Garza, E., \& Caloca, L. (2011). Análisis de la apropiación de las TiC en el aula: más allá de las habilidades tecnológicas. En M. L. López, J. L. López \& J. S. Martínez (Coords.), La comunicación que necesitamos, el país que queremos. XV Encuentro Nacional coneICC. México: Consejo Nacional para la Enseñanza y la Investigación de las Ciencias de la Comunicación. Recuperado de 
http://www.uaeh.edu.mx/investigacion/productos/4915/coneicc.pdf

Lumpkin, A., Achen, R., \& Dodd, R. (2015). Using technology-nested instructional strategies to enhance student learning. Insight: a journal of scholarly teaching, 10, 114-125. Recuperado de http://insightjournal.park.edu/wp-content/ uploads/2015/08/9-Using-Technology-Nested-Instructional-Strategies-toEnhance-Student-Learning.pdf

Martín-Laborda, R. (2005). Las nuevas tecnologías en la educación (Cuadernos de la información 5). Madrid: Fundación AUNA. Recuperado de http://www.telecentros.info/pdfs/05_06 05 tec edu.pdf

Massone, A., \& González, G. (2005). Lectura: comprensión vs. retención de información. Una interpretación cognitiva. Revista Iberoamericana de Educación. Recuperado de http://rieoei.org/didacticag.htm

Monereo, C. (2009). Competencia digital: para qué, quién, dónde y cómo debe enseñarse. Revista Aula de Innovación Educativa, 181, 9-12.

Monereo, C. (Coord.), Castelló, M., Clariana, M., Palma, M., \& Pérez, M. L. (1994). Estrategias de enseñanza y aprendizaje. Barcelona: Graó.

Organización de las Naciones Unidas para la Educación, la Ciencia y la Cultura. (Mayo, 2011). Digital literacy in education. Recuperado de http://unesdoc. unesco.org/images/o021/002144/214485e.pdf

Organización para la Cooperación y el Desarrollo Económicos. (2016). Programa para la Evaluación Internacional de Alumnos (PISA): PISA 2105 Resultados. Recuperado de https://www.oecd.org/pisa/PISA-2015-Mexico-ESP.pdf

Ontoria, A., Ballesteros, A., Cuevas, C., Giraldo, L., Martín, I., Molina, A., Rodríguez, A., \& Vélez, U. (2000). Mapas conceptuales: una técnica para aprender (10 $\mathrm{ed}$.). Madrid: Narcea.

Salinas, J. (Coord.). (2008). Innovación educativa y uso de las TIC. Sevilla: Universidad Internacional de Andalucía. Recuperado de http://dspace.unia.es/bitstream/ handle/10334/2524/innovacioneduc2008.pdf? sequence $=1$

Schneider, M., \& Stern, E. (2010). The cognitive perspective on learning: Ten cornerstone findings. En Organization for Economic Co-Operation and Development (OECD) (Ed.), The nature of learning using research to inspire practice. Recuperado de https://www.researchgate.net/publication/260389966 The cognitive_perspective_on_learning_Ten_cornerstone findings

Talbot, C. (2003). Estudiar a distancia: una guía para estudiantes. Barcelona: Gedisa. Tuckman, B., \& Monetti, D. (2011). Psicología educativa. México: Cengage Learning. 